
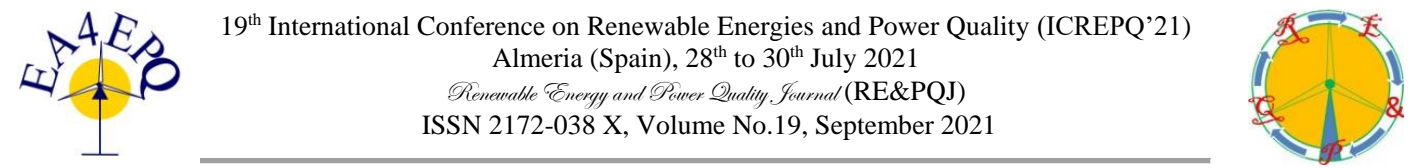

\title{
Distribution Grid Future Planning Under Uncertainty Conditions
}

\author{
V. Samoylenko ${ }^{1}$, A. Firsov ${ }^{1}$, A. Pazderin ${ }^{1}$ and P. Ilyushin ${ }^{2}$ \\ ${ }^{1}$ Department of Automated Electrical Systems \\ Ural Federal University \\ 6200002, Yekaterinburg (Russia) \\ Phone/Fax number:+007 343 3591615, e-mail: v.o.samoylenko@urfu.ru, cap1996@mail.ru, a.v.pazderin@urfu.ru \\ ${ }^{2}$ Energy Research Institute \\ Russian Academy of Sciences \\ 117186, Moscow (Russia) \\ Phone/Fax number:+007 499 1274664, e-mail: ilyushin.pv@mail.ru
}

\begin{abstract}
The paper presents an approach for making decisions about the future development of a distribution grid under uncertainty conditions. The levels of a grid hosting capacity and adequacy are examined using probabilistic approach compared to the conventional deterministic fit-andforget approach. It is shown that the probabilistic approach according to the $99 \%$ confidence probability saves significant costs in comparison with the deterministic approach. The probabilistic calculations prove the use of an equipment rated capacity downsized by 2 points of a typical IEC scale, and in some cases to refuse the construction of a parallel circuit.
\end{abstract}

The main contribution of the paper is a method for choosing an effective rated voltage of a distribution grid in a probabilistic interpretation based on the conventional formulas of Still, Zalessky and Illarionov. The technique includes obtaining the probability of loads location at different distances from power supply centre and the probability of load power distribution in a given range of values. It is shown that the calculation using the developed method makes possible to prefer grid rated voltage at least 1 point downsized by IEC scale with sufficient savings due to the difference in the equipment price compared with the deterministic fit-and-forget approach.

Key words. Distribution grid, distributed energy resources, future planning, decision making, uncertainty conditions.

\section{Introduction}

The issue of distribution grid planning is of great importance nowadays. Due to the development of microgrids and Smart Grids conventional approaches to be revised to ensure proper level of grid adequacy and economy. The main challenge for the planning is a high degree of uncertainty provided by distributed energy sources, including the renewables [1].

The existing design and planning practice for distribution grid future development typically includes a selection of a grid equipment with a significant margin of capacity reserve based on a calculation of peak-load steady state
[2], [3]. This conventional approach is deterministic fitand-forget approach. The margin of reserve often leads to unreasonably high capital investments to a grid, since corresponding high levels of load and generation imbalances that very seldom occur [4]. Historically, this was due non-smart stage of medium-voltage distribution grids development: unidirectional power flows, lack of proper observability of the distribution network, lack of dispatch control (in fact, only service and maintenance were applicated) [5].

A probabilistic approach to the future development of an electrical distribution grid provides avoiding unreasonably high capital investments to a grid during the conditions of increasing load density, increasing length of a grid, and interconnecting distributed generation of all types [6], [7].

The paper presents an approach to making decisions about the future development of a distribution grid under uncertainty conditions. The levels of electrical loads and generation based on renewable energy sources, including photovoltaic and wind generation, are considered as uncertainties. The calculation is performed by a probabilistic method using mathematical distributions describing the relevant influencing factors.

The paper provides the quantative representation of the economy provided by the choise between some rated voltage of a grid given the variety of state and topology diversity. However, the main contribution of the paper is a rated voltage selection for distribution grid based on the probabilistic approach applied to conventional formulas describing the relations between grid load and its length.

\section{Theoretical Background}

The balances depend upon nodal power injections. The nodal injections are represented by loads and sources, like distributed power generation. The idea of the alghorithm is to obtain flows between nodes given the nodal injections with respect to their probability parameters and 
to compare the flows values with the capacity of planned equipment [8]. The stages of calculation are represented futher.

\section{A. Obtaining Nodal Power Distribution Probabilities}

Load power $P$ can be represented by Gaussian distribution [9], [10]:

$$
f(P)=\frac{1}{\sigma_{P} \sqrt{2 \pi}} \exp \left(-\frac{\left(P-P_{m e d}\right)^{2}}{2 \sigma_{P}^{2}}\right)
$$

$P$ is not a constant here; $P_{\text {med }}$ can be represented as medium estimated load in a node; $\sigma_{P}$ can be obtained as the difference between maximum $P_{\max }$ and medium $P_{\text {med }}$ estimated loads from Gaussian distribution properties:

$$
\sigma_{P}=\left(P_{\text {max }}-P_{\text {med }}\right) / 5
$$

It is well known that $\pm 2 \sigma_{P}$ confidence interval corresponds to the $95.4 \%$ confidence probability and $\pm 3 \sigma_{P}$ confidence interval corresponds to the $99.2 \%$ confidence probability.

Wind speed $V$ distribution is described by Weibull probability distribution [11], [12]:

$$
f(V)=\frac{k}{\lambda}\left(\frac{V}{\lambda}\right)^{k-1} \exp \left(-\left(\frac{V}{\lambda}\right)^{k}\right)
$$

where $k$ and $\lambda$ are probability distribution parameters obtained from gathered statistics or numerical weather prediction for a given region where the grid is located.

To convert wind speed to wind power wind-to-power curve $P_{\text {turb }}=f(V)$ is used according to IEC 61400 [13] for each wind turbine. For on-shore wind farms IIIa and IIIb wind classes are the most common. IEC IIIb class is used in this work for all the wind farms. So, the resulting distribution of a wind power is represented as:

$$
P_{W}(V)=f(V) \cdot P_{t u r b}(V)
$$

PV power is modelled by beta distribution [14]-[16]:

$$
f(P)=\frac{P^{\alpha-1}(1-P)^{\beta-1}}{B(\alpha, \beta)}, \quad B(\alpha, \beta)=\int_{0}^{1} P^{\alpha-1}(1-P)^{\beta-1},
$$

where $\alpha$ and $\beta$ are probability distribution parameters obtained from gathered statistics for a given region where a distribution grid is located.

After obtaining nodal power distribution probability density functions according to engineering practise it is useful to convert them into numerical integration-friendly format. The idea is to divide the distribution into stepped form graph with the square of rectangle at each step equal to the corresponding integral of the replaced probability function (Fig. 1). Discrete representation of probability values provides more efficient calculations at the next step.

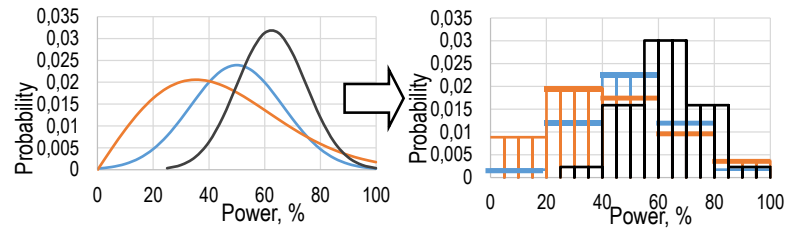

Fig. 1. Probability density graph discretisation. Load is shown in dark grey, wind in orange, and photovoltaic in blue

\section{B. Calculating Different Combinations of Balances}

Calculating of balances (unbalances) is implemented via the calculation of different combinations of nodal power values with respect to their probability. A huge set of literature is devoted to the probabilistic calculation of steady states [17]-[21]. Improving corresponding methods and techniques is out of the scope of this paper. However, the following points to be noted.

One of the main features complicating probabilistic steady state calculation is the covariance (and correlation) between nodal power values leading to the covariance between voltage values. This matters if the calculation is based on voltages [22]. There are two different approaches providing the solution. The first one is the vector space transformation by means of different methods [23]. Taking into account covariance values is an exact approach, although it leads to high computation load, especially in spreading distribution grids.

The second approach is the analysis of the errors arising if the corresponding covariance and correlation between two nodal power values is neglected [22]. A good methodical assumption is the independent voltage control in different nodes that provides smaller error value. For planned future Smart Grids this assumption is close to the reality because inverter-based distributed generation and frequencydriven motors occupying more than $70 \%$ of load that can regulate voltage in the discussed way. Final contribution makes an aggregation of the same-type loads (industrial loads, residential, commercial, etc.). In this case distinguishing loads, as well as distinguishing sources to be supposed low-correlated (independent). During the calculation all the same-type loads are supposed to have the same probability of relative power values.

Both approaches results in an opportunity to use computationally simple and effective combination of nodal power probabilities to calculate the whole set:

$$
\operatorname{Pr}\left\{P_{1} \in S_{1}, P_{n} \in S_{n}\right\}=\prod_{i=1}^{N} P_{i} \in S_{i}
$$

where $N$ is a number of nodes; $S$ is an independent set of particular-type power values, including wind power, photovoltaic power, industrial load power, commercial load power, residential load power, etc. Traditional constant coincidence (simultaneity) factors imply strong mutual correlation between the considered power values and can't provide how often and how long these cases of the simultaneity arise and the total share of the states to be excluded for prospective planning. 
Further calculations can be made using one of the steadystate calculation methods [23]. This work uses current balance-based equations of state parameters. The initial data for the calculation includes power lines conductivity in $\mathrm{S}$, or p.u. along with $\mathrm{km}$ if $R / X$ ratio of a grid is unified. Nodal voltages in current balance form:

$$
\mathbf{Y U}+\mathbf{Y}_{\mathrm{B}} \mathbf{U}_{\mathbf{B}}=\frac{\mathbf{P}}{\mathbf{I}}
$$

where $\mathbf{Y}$ is a matrix of conductivity: $\mathbf{U}, \mathbf{P}, \mathbf{I}$ are vectors of nodal voltages, power values and nodal currents, correspondingly. The results of the calculation are steadystate parameters, including voltages and currents.

\section{Selecting a Set of States for Making Decision}

After calculating state parameters for the whole set of states different probabilistic criteria can be applied to support decision making. The most common criteria to be applied are confidence probability criteria of 95 and $99 \%$ [6]. According to this criteria, $5 \%$ and $1 \%$ of unlikely states are neglected, correspondingly (Fig. 2.)

\section{Distribution Grid Capacity Planning}

The paper examines levels of a grid hosting capacity and adequacy in terms of steady-state parameters (like voltages and currents) satisfying a grid code with probability of $95 \%$ and $99 \%$. The levels are compared to the necessary levels in terms of a deterministic approach of $100 \%$ probability. The set of distribution grids under consideration include $10-35 \mathrm{kV}$ urban and rural grids:

1) urban $10 \mathrm{kV}$ grids made of underground cable lines that typically suffers from overcurrents;

2) rural $10 \mathrm{kV}$ grids made of self-carrying isolated cable and subject to both overcurrents and voltage deviations;

3) rural $35 \mathrm{kV}$ overhead grids made of steel reinforced aluminium wire and subject mostly to voltage deviations.

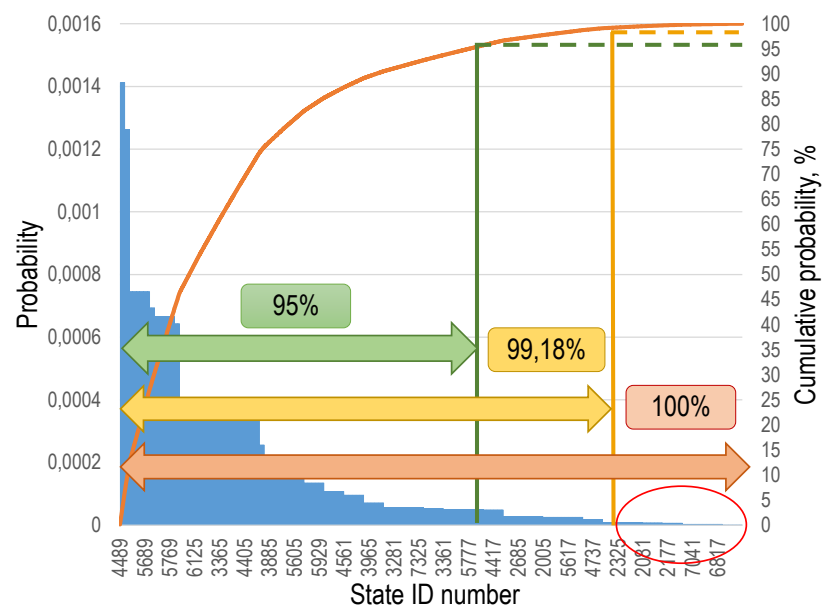

Fig. 2. Selecting a set of states according to confidence probability criteria. Red ellipse circumscribes typical scale of states share that very seldom arise but require additional capacity

Radial-type, loop and trunk topology up to 7 substations in a sequence were considered (Fig. 3). The analysed set includes distinguishing combinations of nodal and generation loads, such as regular, interlaced or consolidated nodal location types. In addition, singlecircuit and double-circuit distribution power lines of a grid are considered. Nodal load and distributed generation maximum values vary randomly from $4 \mathrm{MW}$ to $8 \mathrm{MW}$.

The concept and some results of capacity planning are shown in Fig. 4. Each confidence probability has corresponding confidence interval of power, current and voltage. As it can be seen from the figure, $5 \%$ or $1 \%$ of the most rare states excluded from the set depending upon the confidence probability criteria $95 \%$ and $99 \%$, correspondingly. These excluded states are always represent the most rare states, like maximum load combined with minimum generation, or, vice versa, maximum generation combined with minimum load. These rare states are completely taken into account by fitand-forget deterministic approach, but not by a probabilistic approach. The residual less than $1 \%$ of cases and states followed by overloads and unacceptable voltage drops are considered as pre-emergency and emergency states to be eliminated by means of relay protection and emergency control automation devices.

The results of the economy that arises for the cases of probabilistic approach-based grid development is shown in Table I. The probabilistic calculations prove the use of an equipment rated capacity downsized by 1 point [24] for $99 \%$ confidence probability and up to 2 cross-section points for $95 \%$ confidence probability. Averaged through case study downsize corresponds to the average economy. In case if the required due to fit-and-forget approach cross-section exceeds maximum available for singlecircuit $\left(630,120\right.$ and $150 \mathrm{~mm}^{2}$, correspondingly), the probabilistic approach in some cases provides to refuse the construction of a parallel circuit, like for the case of rural $35 \mathrm{kV}$ grid according to $95 \%$ confidence probability criterion. It corresponds to maximum economy value. In should be noted that the reduction of wire-cross section itself for $35 \mathrm{kV}$ overhead lines provides the economy of $\sim 3 \%$ only. but the main share of economy is due to the utilization of more light overhead line towers and tower bases for light wire of low cross-section.

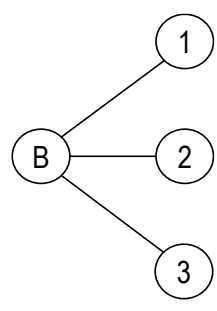

a)

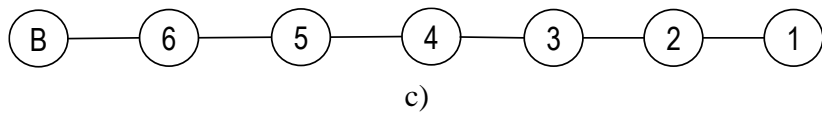

Fig. 3. Considered topologies of a distribution grid: a) radial; b) loop; c) trunk. Sign B (base node) marks power supply centre 


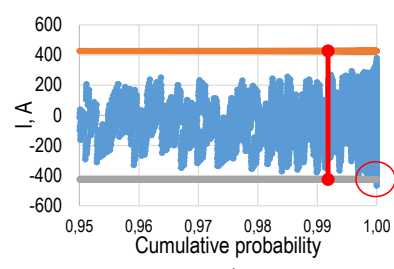

a)

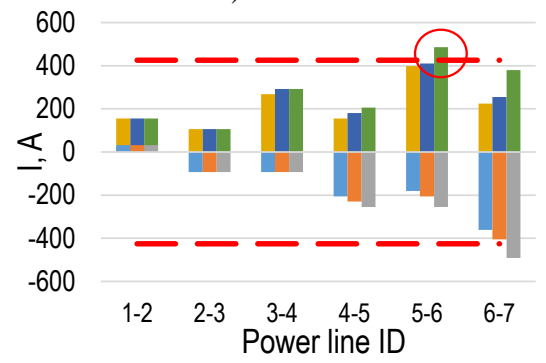

c)

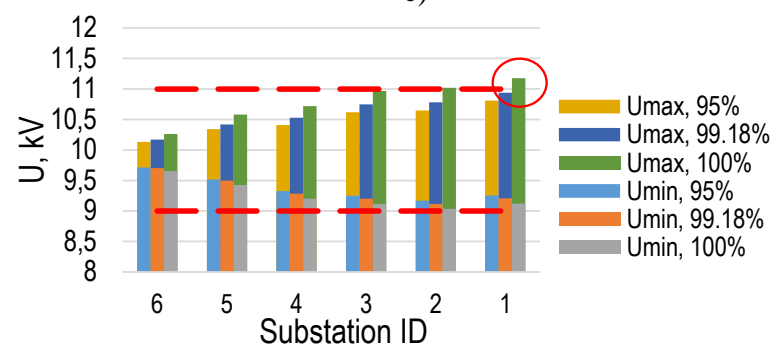

d)

Fig. 4. Probabilistic exclusion of steady states at $10 \mathrm{kV}$ grid shown in Fig. 3, c by means of $95 \%$ and $99 \%$ criteria compared to deterministic approach: a) overcurrent concept for $99 \%$ (minus sign denotes reverse direction of a current); b) overvoltage concept for $99 \%$; c) overcurrent at the power line between substations No. 5 and No. 6; d) overvoltage at the substations No. 1

Table I. - Costs Economy at Distribution Grid Planning by Means of Necessary Cross-Section Reduction

\begin{tabular}{|c|c|c|c|c|}
\hline $\begin{array}{c}\text { Grid } \\
\text { type }\end{array}$ & $\begin{array}{c}\text { Cross- } \\
\text { sections, } \\
\mathrm{mm}^{2}\end{array}$ & $\begin{array}{c}\text { Confidence } \\
\text { probability } \\
\text { applied }\end{array}$ & $\begin{array}{c}\text { Cross- } \\
\text { section } \\
\text { reduction }\end{array}$ & $\begin{array}{c}\text { Costs economy, } \\
\text { average/max, \% }\end{array}$ \\
\hline Urban & 120,240, & $95 \%$ & 500 to 300 & $14.4 / 20.6$ \\
\cline { 3 - 5 } $10 \mathrm{kV}$ & $300,400,630$ & $99 \%$ & 500 to 400 & $11.3 / 15.2$ \\
\hline Rural & 70,95, & $95 \%$ & 120 to 70 & $10.1 / 16.8$ \\
\cline { 3 - 5 } $10 \mathrm{kV}$ & 120 & $99 \%$ & 120 to 95 & $7.3 / 11.3$ \\
\hline Rural & 70,95, & $95 \%$ & 150 to 95 & $21.6 / 30.9$ \\
\cline { 3 - 5 } $35 \mathrm{kV}$ & 120,150 & $99 \%$ & 150 to 120 & $12.1 / 22.7$ \\
\hline
\end{tabular}

\section{Distribution Grid Rated Voltage Selection}

Although probabilistic approach provides some amount of costs economy for a distribution grid during capacity selection, the real potential for costs economy is a rated voltage selection. In real practise there is always a number of power supply centres that are available for a new distribution grid interconnection and development [1], [6]. The question is what rated voltage is more preferable to ensure efficient power distribution.

IEC standard scale assumes rated voltage is a discrete value [25]. Furthermore, in many countries according to grid codes there is a great gap between two adjacent rated voltages, for example, $6-20-110 \mathrm{kV}$ or $10-35-110 \mathrm{kV}$. In this case the selection of a proper rated voltage is of great performance, because an overestimation leads to sufficient cost overrun, due to the fact that grid equipment will hardly ever operate at the rated load levels.

There is a number of empirical formulas describing the dependency between power, power line length and required voltage. Well-known formulas that are used in different countries worldwide are Still formula, Illarionov formula and Zalessky formulas [26]. The paper presents a method for choosing an effective rated voltage of a distribution grid in a probabilistic interpretation based on these conventional formulas.

Still formula is written as:

$$
U_{\text {rated }}=4.34 \sqrt{D+16 P},
$$

where $D$ is a distribution distance in $\mathrm{km}$; $P$ is a power in MW to be conducted. There is another one representation of the formula: $U_{\text {rated }}=5.5 \sqrt{ }(0.62 D+3 P / 100)=$ $4.33 \sqrt{ }(D+48.3 P)$. The difference is that this formulation calls for a single-phase power value, so rated voltage can be selected for double-phase or single-phase lines too.

Illarionov formula is known as:

$$
U_{\text {rated }}=\frac{1000}{\sqrt{\frac{500}{D}+\frac{2500}{P}}}
$$

Zalessky formula is represented by:

$$
U_{\text {rated }}=\sqrt{P(100+15 \sqrt{D})}
$$

Accounting power in the formulas is made in the same way as it described in Chapters 2 and 3 of the paper. The power of load is supposed not to exceed the upper border of the confidence interval corresponding to $95 \%$ or $99 \%$ confidence probability depending on the criterion applied.

Load can be located at the different distance from power supply centre of distribution grid. Furthermore, in general load can exist with a distinguishing probability. The calculation of a distance is a standard square root of 2 coordinates (if height difference is not too large to be neglected). Nevertheless, a matching between distance and probability is required. The method proposed provides for a calculation of a distance by means of a cellular principle. The planed area of future distribution grid is divided to square cells with a power supply center located at the centre of the planned area. All the squares are filled with probability shares that form 1.000 for the area under consideration. The case study illustrates rural area, besides in urban areas all the distances are smaller.

The source values of a distance to be defined by means of a map of planned objects. Probability can be defined by some expert methods [17], but in this work probability is defined as the share of the total base area of the objects that corresponds to $m$-th square cellar of a grid area: 


$$
\sum_{m=1}^{M} p_{m}=\sum_{i=m}^{M} \frac{A_{o b j . m}}{A_{o b j}}=1
$$

where $M$ is a number of square cells, $A_{o b j . m}$ is a summary area of the objects located at $m$-th cell and $A_{o b j}$ is a total square of the objects to be supplied by the grid. Calculation of a distance is shown in Fig. 5.

Different combinations of load power probability and location probability can be obtained. They can be interpreted as follows:

1) Low probability of load existence, low load power according to $95 \%$ and $99 \%$ criteria. This means that it's unlikely to find a load located in the point. Examples include urban park area;

2) Low probability of load existence, high load power according to $95 \%$ and $99 \%$ criteria. This means that exact load location is under consideration due to some environmental restrictions, like new rural industrial plant layout and city office district internal planning;

3) High probability of load existence, low load power according to $95 \%$ and $99 \%$ criteria. This corresponds to some minor secondary objects, like industrial auxiliary or commercial buildings;

4) High probability of load existence, high load power according to $95 \%$ and $99 \%$ criteria. This corresponds to major primary objects, like industrial core or residential community.

A case study based on the map shown in Fig. 5. Nodal power values varying from $5 \mathrm{MW}$ to $25 \mathrm{MW}$ in a random order were conducted. The results of rated voltage calculation according to formulas are shown in Fig. 6.

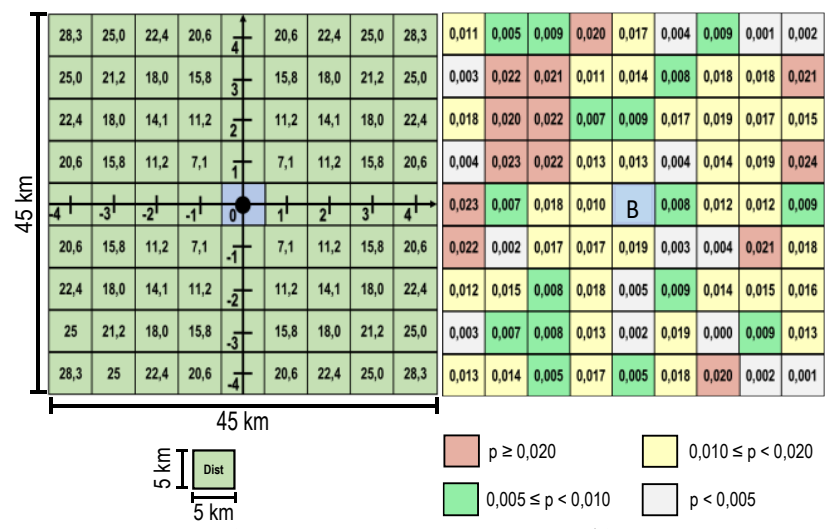

a)

b)

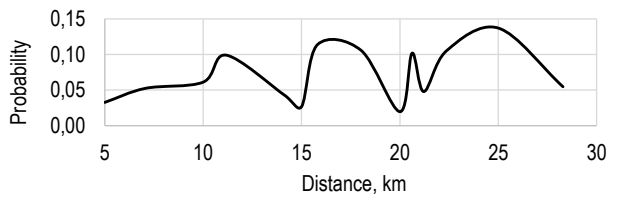

c)

Fig. 5. Probability of load allocation in a distribution grid area using a case study of rural grid with mining, farm and village loads: a) square cellular principle with the values of distances from power supply center; b) probabilities; c) probability as a function of a distance. Sign B denotes power supply center
Different metrics and estimations could be applied for the selection of rated voltage. First, reference value to compare with that can be determined as:

1) simple average between rated voltages $(72.5 \mathrm{kV}$ in the case study between $35 \mathrm{kV}$ and $110 \mathrm{kV})$;

2) taking into account voltage against power nonlinearity, root mean square between rated voltages $(81.6 \mathrm{kV})$;

3) equal costs value for the rated voltages (depends upon the country).

Secondly, the metrics for estimating the obtained dataset may vary. Essential metrics include weighted by probability rated voltage $\Sigma p_{i} U_{\text {rated. } i}$ (used further in the work), averaged via the dataset or median value. Furthermore, for the deterministic fit-and-forget approach rated voltage to be defined by means of the maximum value of the dataset regardless of the uncertainty either in load value or in load distance allocation. The results of rated voltage selection is shown in Table II. Based on the majority of estimations, $35 \mathrm{kV}$ rated voltage can be selected for the distribution grid.

Specific costs for $35 \mathrm{kV}$ grid construction and operation compared to $110 \mathrm{kV}$ ones depend upon the region, but for this work they are $41 \%$ lower. That states a great potential for costs economy using the proposed approach and methods.

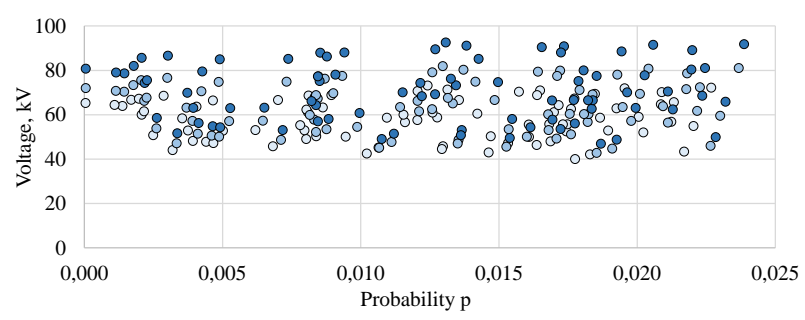

a) Results for Still formula

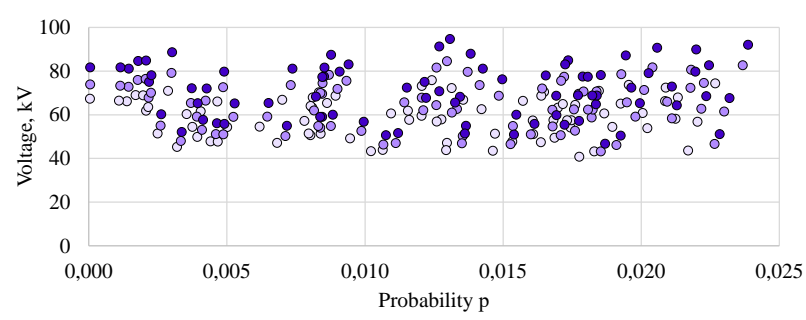

b) Results for Illarionov formula

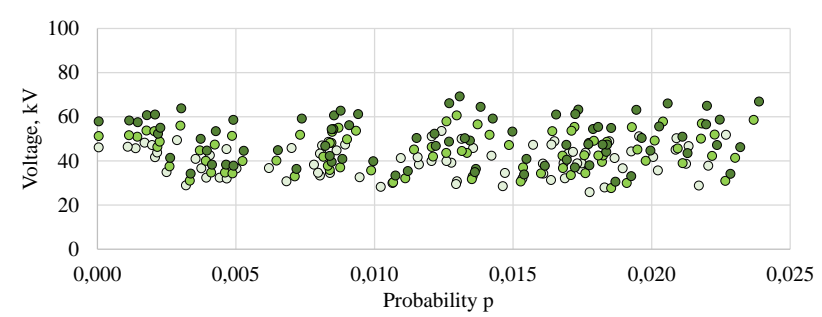

c) Results for Zalessky formula

Fig. 6. Appropriate rated voltage according to the formulas. Vertical axis denotes recommended voltage in a variety of steady states. Horizontal axis denotes the probability of each state (the sum is 1.000). Light coloured dots denote $95 \%$ confidence interval, medium coloured dots denote $99 \%$ confidence interval and dark saturated dots denote deterministic approach 
Table II. - Rated Voltage Selection

\begin{tabular}{|l|c|c|c|}
\hline \multirow{2}{*}{$\begin{array}{l}\text { Load power } \\
\text { confidential } \\
\text { probability }\end{array}$} & \multicolumn{3}{|c|}{ Rated voltage selection by formula, $\mathrm{kV}$} \\
\cline { 2 - 4 } & Still & Illarionov & Zalessky \\
\hline $95 \%$ & $52.6 \rightarrow 35$ & $56.4 \rightarrow 35$ & $35.9 \rightarrow 35$ \\
\hline $99 \%$ & $63.3 \rightarrow 35$ & $65.2 \rightarrow 35$ & $42.6 \rightarrow 35$ \\
\hline $100 \%$ & $92.6 \rightarrow 110$ & $94.8 \rightarrow 110$ & $73.2 \rightarrow 110$ \\
\hline
\end{tabular}

\section{Conclusion}

The proposed methods provide the following possibilities:

1) To carry out the selection of probable grid operation steady states according to the given confidential probability criteria.

2) To select hosting capacity providing costs economy of 7.3-30.9\% for $10-35 \mathrm{kV}$ distribution grids compared to deterministic approach;

3) To select rated voltage of a distribution grid based on the probabilistic approach to conventional formulas, that provides an economy up to $41 \%$ (depends upon world region).

Further investigations to be concentrated on uncertainty analysis and on metrics for rated voltage estimations.

\section{Acknowledgement}

Proposed results are the outcome of work on the topic "Development and testing algorithms and technical solutions for digital control of power facilities" within integrated project "Improvement of approaches to digital management of power facilities".

\section{References}

[1] F. Pilo, "How the clean energy for all Europeans package will reshape planning and development strategies in power distribution", 2019 IEEE International Conference on Environment and Electrical Engineering and 2019 IEEE Industrial and Commercial Power Systems Europe (2019). pp. 0-21

[2] G. D'Antona, “Uncertainty Of Power System State Estimates Due To Measurements And Network Parameter Uncertainty" 2010 IEEE International Workshop on Applied Measurements for Power Systems (2010). pp. 0-5

[3] T. Whalen, "Propagation Of Uncertainty In Systems With Both Probabilistic And Possibilistic Inputs", 2014 IEEE Conference on Norbert Wiener in the 21st Century (2014), pp. 0-5

[4] S. A. Rashidaee, T. Amraee, "Generation Expansion Planning Considering the Uncertainty of Yearly Peak Loads", 2018 IEEE International Conference on Environment and Electrical Engineering and 2018 IEEE Industrial and Commercial Power Systems Europe (2018). pp. $0-4$

[5] G. Celli, L.Schiavo and F. Pilo et al., "Assessment of Energy Storage Systems Installation in Smart Distribution Networks". AEIT International Annual Conference (2018). pp. 0-5.

[6] F. Pilo, Planning of Distribution Systems in the Era of Smart Grids, ISGAN Webinar (2018). 59 ps.

[7] F. Pilo, G. Pisano and G. Soma, "Digital Model Of A Distribution Management System For The Optimal
Operation Of Active Distributions Systems", CIRED Seminar: SmartGrids for Distribution (2008). pp. 0-4.

[8] S. Kannan and M. Teng, "Probabilistic approach in sizing distribution transformers", IEEE 11th International Conference on Probabilistic Methods Applied to Power Systems (2010). pp. 0-4.

[9] A. Ahmad, I. Azmira, S. Ahmad, "Statistical distributions of load profiling data", IEEE International Power Engineering and Optimization Conference (2012). pp. 199203

[10] T. Zhang, W. Shen and X. Song et al., "Probabilistic Modelling and Simulation of Stochastic Load for Power System Studies", UKSim 15th International Conference on Computer Modelling and Simulation (2013). pp. 519-524

[11] V. Katinas, G. Gecevicius and M. Marciukaitis, "An investigation of wind power density distribution at location with low and high wind speeds using statistical model", Applied energy (2018). Vol. 3, pp. 442-451

[12] P. Wais, "Two and three-parameter Weibull distribution in available wind power analysis", Renewable Energy (2017), Vol. 103, pp. 15-29.

[13] IEC 61400-12-1:2005 Power performance measurements of electricity producing wind turbines. IEC, 2005.

[14] S. Trachenkov and V. Astapov, "The The Applicability of Zero Inflated Beta Distributions for Stochastic Modeling of PV Plants' Power Output", 19th International Scientific Conference on Electric Power Engineering (2018). pp. 0-5.

[15] A. Guwaeder and R. Ramakumar, "Statistical Analysis of PV Insolation Data", IEEE 44th Photovoltaic Specialist Conference (2017). pp. 0-3.

[16] K. Ashenayi, S. Singh and I. Hoballah, "Application of normal distribution in modeling global irradiation", The Twentieth Southeastern Symposium on System Theory (1988) pp. 470-474

[17] C. N. Ning, C. A. Hsieh and T. Y. Hsiao et al., "Two Application Examples Of Probabilistic Risk Assessment In Power System Operations", International Conference on Probabilistic Methods Applied to Power Systems (2006). pp. 0-4.

[18] N. Abdel-Karim, D. Calderon and M. Lauby et al., 'Impact of wind and solar variability on the resource adequacy for North American bulk power system", International Conference on Probabilistic Methods Applied to Power Systems (2016), pp. 0-7.

[19] H. Quan, D. Yang, A. M. Khambadkone and D. Srinivasan, "A Stochastic Power Flow Study to Investigate the Effects of Renewable Energy". IEEE Innovative Smart Grid Technologies - Asia (2018). Vol. 1, pp. 19-24

[20] Y. Lin-Hao, "Research on Voltage of Distribution Networks with Distributed Photovoltaic System based on Probabilistic Model", IEEE 2nd International Future Energy Electronics Conference (2015), pp. 0-5

[21] G. Shi, S. Eftekharnejad, "Impact of solar forecasting on power system planning", North American Power Symposium (2016) pp. 0-5

[22] M. B. Maskar, A. R. Thorat and P. D. Bamaneet al., "Optimal power flow incorporating thermal and wind power plant", International Conference on Circuit, Power and Computing Technologies (2017). pp. 0-5

[23] H. Yang, R. Qiu, and L. Chu et al., "Improving Power System State Estimation Based on Matrix-Level Cleaning", IEEE Transactions on Power Systems (2020), Vol. 35, pp. 3529-3540.

[24] IEC 60228:2004 Conductors of insulated cables. IEC, 2004.

[25] IEC 60038:2009. IEC standard voltages. IEC, 2009.

[26] G.N. Klimova, E.A. Shutov,I.V. and Sharapova, Industrial Energy Efficiency, Tomsk, TPU Publishing House, 2015. $163 \mathrm{ps}$. 